\title{
Alcoholysis of palm oil mid-fraction by lipase from Rhizopus rhizopodiformis
}

\begin{abstract}
A mycelial lipase from Rhizopus rhizopodiformis was prepared in fragment form. The lipase was examined to catalyze the alcoholysis of palm oil mid-fraction (PMF) in organic solvents. High percentage conversions of PMF to alkyl esters were achieved when methanol or propanol was used as acyl acceptor. Of the two most prevalent fatty acids in PMF, palmitic acid seemed to be preferred over oleic acid in the formation of methyl and propyl esters. The optimal ratio of oil to methanol in the alcoholysis reaction is 1 to 2 moles. The lipase exhibited high alcoholysis activities in nonpolar solvents $(\log \mathrm{P}>2)$, such as hexane, benzene, toluene, and heptane. The enzyme showed exceptionally high thermostability.
\end{abstract}

Keyword: Alcoholysis; Mycelial lipase; Solvents; Stability 